\title{
Multifocal osteonecrosis affecting all four limbs in systemic lupus erythematosus: A case report
}

\author{
SIQIAO SUN ${ }^{1}$, QI WANG ${ }^{1}$, YUBAO GONG $^{2}, \mathrm{XIN} \mathrm{QI}^{2}$ and JIANGUO LIU ${ }^{2}$ \\ Departments of ${ }^{1}$ Vascular Surgery and ${ }^{2}$ Bone and Joint Surgery, \\ The First Hospital of Jilin University, Jilin, Changchun 130021, P.R. China
}

Received February 9, 2015; Accepted June 29, 2018

DOI: $10.3892 /$ etm.2019.7829

\begin{abstract}
Osteonecrosis is a condition that frequently affects patients with systemic lupus erythematosus (SLE). However, reports on severe multifocal osteonecrosis involving more than three anatomical sites in patients with SLE are scarce. The present study describes a case of SLE with multifocal osteonecrosis involving all four limbs. A 22-year-old woman was diagnosed with SLE in 2010 and the disease was controlled by treatment with methylprednisolone and hydroxychloroquine. Approximately 1 year following the diagnosis of SLE, the patient developed pain in the elbows and hips. Magnetic resonance imaging revealed evidence of multifocal osteonecrosis in the bilateral distal segments of the humerus and femoral head, the distal segment of the right femur and the proximal segment of the right tibia. The patient was treated with right total hip arthroplasty and core decompression of the right femur and tibia, followed by continuation of the earlier medical treatment for SLE. The present case highlights the importance of conducting a careful investigation of patients with SLE who present with multiple joint involvement, and the choice of the appropriate treatment according to the presentation.
\end{abstract}

\section{Introduction}

Osteonecrosis is defined as necrosis of the bone marrow and trabecular bones due to the lack of sufficient blood supply (1). The occurrence of osteonecrosis in patients with systemic lupus erythematosus (SLE) was first reported in 1960 (2) and has been frequently reported since, with an incidence ranging from 3 to $30 \%$ (3). Many risk factors have been reported to be involved in the development of osteonecrosis in patients with SLE, including trauma a history of autoimmune connective tissue diseases, renal diseases,

Correspondence to: Dr Yubao Gong, Department of Bone and Joint Surgery, The First Hospital of Jilin University, 71 Xinmin Street, Jilin, Changchun 130021, P.R. China

E-mail: gongyubao@hotmail.com

Key words: bone, necrosis, total hip arthroplasty, methylprednisolone, systemic lupus erythematosus coagulation disorders, other hematological disorders, metabolic disorders, infections, alcohol abuse, smoking and the use of corticosteroids or cytotoxic drugs (1). Since asymptomatic osteonecrosis may remain undetected, its true prevalence could be much higher than reported. The estimation of the overall incidence rate of osteonecrosis in SLE patients is misleading as patients are mostly treated with glucocorticoids, which may also result in the development of osteonecrosis. A previous Meta-analysis demonstrates a significant increased risk of osteonecrosis in SLE patients with high disease activity scores (4). Although osteonecrosis is frequently reported in cases of SLE, multifocal lesions involving more than three anatomical sites are unusual (5-16). In the present case report, a case of multifocal necrosis affecting all limbs of a patient with SLE is described.

\section{Case report}

The patient was a 22-year-old woman diagnosed with SLE in June 2010 at The Second Hospital Affiliated to Jilin University and confirmed at the Third Hospital Affiliated to Jilin University. The diagnosis of SLE was made according to the following symptoms and laboratory tests: Fever of unknown origin, malar rash, immunologic disorder and positive antinuclear antibodies. Following diagnosis, the patient received regular oral treatment with $12 \mathrm{mg}$ /day methylprednisolone and $200 \mathrm{mg} /$ day hydroxychloroquine, and the disease was initially well controlled by monitoring immunologic function via immunity tests including turbidinetric inhibition immunoassay (including Immunoglobulin ( Ig)G, Ig A, IgM, $\mathrm{C} 3$ and $\mathrm{C} 4$ ) and assessing the levels of antinuclear antibodies. The disease is considered to be controlled when serum levels of immunoglobin and complement return to normal levels. However, in February 2011, the patient reported bilateral pain and restricted hip movement, and 4 months later, the patient developed similar symptoms in the elbows. Plain radiographs of the elbows indicated bilateral osteonecrosis in the distal segments of the humerus and femoral heads (Fig. 1A). In February 2012, the patient developed pain in the right knee. Due to considerable worsening of the symptoms, the patient was hospitalized on May 29, 2012. On examination, the patient's hip movement was observed to be severely restricted. For the right and left hips, the range of movement was 120 


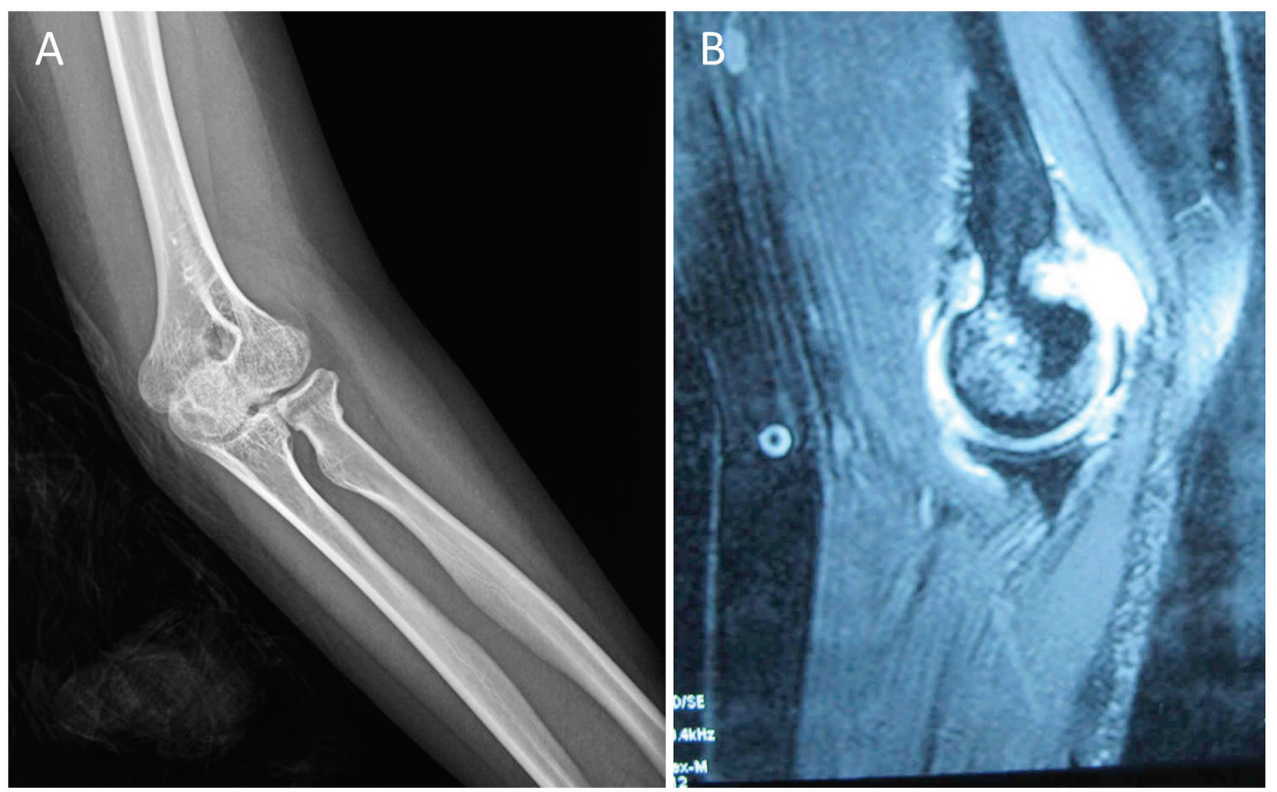

Figure 1. (A) Radiograph of the left distal segment of the humerus exhibits signs of osteonecrosis. (B) Magnetic resonance image reveals osteonecrosis in the left distal segment of the humerus.

and $130^{\circ}$ for flexion; 30 and $40^{\circ}$ for adduction; 20 and $30^{\circ}$ for abduction; and $0^{\circ}$ for extension, pronation and supination, respectively. Furthermore, the range of movement in the left and right elbows was $35-155^{\circ}$ and $25-160^{\circ}$, respectively. Magnetic resonance imaging (MRI) revealed signs of multifocal osteonecrosis in the distal segments of the humerus and femoral heads bilaterally, and in the distal segment of the femur and the proximal segment of the tibia on the right side of the patient (Fig. 1B). No evidence of narrowing of the joint space or damage to the joint surface was observed in the elbows or knee joints. Laboratory tests of antinuclear antibodies were performed using an indirect immunofluorescence assay (17), which revealed that the patient was highly positive for anti-Ro52, anti-Sjögren's syndrome type A and anti-nuclear ribonucleoprotein/Sm antibodies. Furthermore, the level of hypersensitive C-reactive protein was $1.36 \mathrm{mg} / \mathrm{l}$ (normal range, $0-3.5 \mathrm{mg} / \mathrm{l})$, the erythrocyte sedimentation rate was mildly elevated at $38 \mathrm{~mm} / \mathrm{h}$, (normal range, $0-15 \mathrm{~mm} / \mathrm{h}$ ) and the serum complement $\mathrm{C} 3$ and $\mathrm{C} 4$ levels were $1.0 \mathrm{~g} / 1$ (normal range, 0.9-1.8 g/l) and 0.2 g/l (normal range, 0.1-0.4 g/l), respectively. On June 4, 2012, the patient underwent total hip arthroplasty of the right side and drilling decompression of the right femur and tibia. The surgery was successful and recovery was uneventful. Thereafter, the patient's previous treatment was continued, and the patient has remained stable since the surgery. The patient was followed up every 6 months at the Department of Rheumatology and Immunology of the First Hospital of Jilin University (Jilin, China). The condition of SLE was stable according to the results of immune tests. Multiple bone infarction and total hip replacement was monitored at the Department of Bone and Joint Surgery of the First Hospital of Jilin University (Jilin, China) once a year, with no apparent pain symptoms being reported during follow-up. The last follow-up was performed in April 2018. Informed consent was obtained from the patient for the report of the present case.

\section{Discussion}

Osteonecrosis is characterized by the death of bone marrow and trabecular bone tissue due to disruption of the blood supply to bone tissues (2). SLE is the most common rheumatic disease that causes osteonecrosis (18). Traumatic causes of osteonecrosis may lead to the displacement of bone fragments, resulting in impaired blood supply and ischemia of the affected bone tissue (19). However, when osteonecrosis occurs due to systemic disease, the pathogenesis involves disruption of the blood supply to the bone, which leads to a sequence of events, including localized hyperemia, demineralization, trabecular thinning and collapse of the bony architecture (20). Bone infarcts usually remain clinically asymptomatic and so are frequently only recognized by characteristic radiographic changes in the later stages, which include dense, irregular shadows resembling puffs of smoke in the metaphyso-diaphyseal regions of the long bones (20). MRI examination is helpful in such cases since it enables the early detection of bone infarcts; these are observed as areas of low signal intensity with irregular margins and dispersed areas of increased signal intensity that indicate the presence of fat (21). In the present case, radiographic examination was performed for the evaluation of the initial symptoms of joint pain. The results showed a slightly increase in density and osteonecrosis was under suspicion. Subsequent MRI examination provided definitive information that helped to establish the diagnosis of osteonecrosis.

The risk factors implicated in the development of osteonecrosis in patients with SLE include trauma and a history of autoimmune connective tissue diseases, renal diseases, coagulation and other hematological disorders, metabolic disorders, infections, alcohol abuse, smoking, and the use of corticosteroids and cytotoxic drugs (1). With regard to the pathogenesis of multifocal osteonecrosis in SLE, the use of corticosteroids and cytotoxic drugs is the most relevant factor (19). The 
association between osteonecrosis and the use of corticosteroids is evident from the observation that the condition is common in patients with SLE who have been treated with corticosteroids and extremely rare in those naïve to corticosteroid use $(22,23)$. Notably, previous studies have shown that the duration, total cumulative dose and highest daily dose of corticosteroids are independent factors associated with the occurrence of osteonecrosis (24-26). In the present case, the development of multifocal osteonecrosis may be attributed to the administration of high-dose methylprednisolone for a period of more than 1 year.

The anti-inflammatory and immunosuppressive effects of corticosteroids are mediated via reductions in the expression of cytokines and adhesion molecules; inhibition of the migration and access of leukocytes to the site of inflammation; and disruption of the functioning of leukocytes, fibroblasts and endothelial cells (27). In addition, corticosteroids inhibit osteoblastic activity, leading to decreased bone formation, loss of bone mineral density and increased risk of fracture (28). Bone loss begins when corticosteroid treatment is initiated and peaks at 6 months of therapy; it decreases when corticosteroid use is discontinued (28). Loss of bone tissue mainly occurs in regions with a high content of trabecular tissue, such as the vertebrae (29). Corticosteroids may increase the size and number of intramedullary lipocytes; this raises the pressure within the bone marrow, which results in vascular compression and reduced perfusion of the bone tissue, thereby triggering bone ischemia and infarct (30). Evidence supporting this hypothesis has been obtained from MRI study, which has shown that compared with healthy controls, patients with SLE have a higher percentage of bone-marrow fat and exhibit a higher degree of conversion of hematopoietic marrow into fatty bone marrow (31). The percentage of fatty marrow has been demonstrated to correlate with the mean daily dose of corticosteroids and the presence of osteonecrosis (31). Sequential MRI scans of patients with SLE and osteonecrosis have indicated the presence of extensive bone-marrow edema (32). This is further supported by the evident relief of the symptoms of osteonecrosis following core decompression.

The treatment of osteonecrosis in patients with SLE is similar to that of osteonecrosis in general, as is the outcome of the treatment. The main therapeutic approaches for osteonecrosis are medical management and surgery $(22,23)$. Small and asymptomatic lesions may heal spontaneously or remain stable without progressing or causing significant joint damage. However, symptomatic lesions require a combined approach involving medical and surgical management. The medical management of osteonecrosis comprises the administration of analgesics, physical therapy, and the use of devices to prevent muscle atrophy and allow non-weight bearing movement $(23,32)$. Cases in which osteonecrosis affects the femoral heads often require total hip arthroplasty $(22,23)$, as in the present case. Another widely used approach in the management of the early stages of osteonecrosis is core decompression, which aims to reduce the intramedullary pressure at the osteonecrotic lesions, thereby improving perfusion of the ischemic bone (33). In the current case, core decompression was performed for the right femur and tibia, where the lesions were less severe.
In conclusion, the present case highlights the importance of conducting a thorough investigation for multifocal osteonecrosis in patients with SLE and pain in multiple joints. On the basis of the present case, MRI is suggested to be the diagnostic modality of choice for osteonecrosis and more informative than radiography.

\section{Acknowledgements}

Not applicable.

\section{Funding}

No funding was received.

\section{Availability of data and materials}

The datasets used and/or analyzed during the present study are available from the corresponding author on reasonable request.

\section{Authors' contributions}

SS acquired the data and wrote the manuscript; QW analysed and interpreted the data, and revised the manuscript; YG collected the data, and conceived and supervised the study; XQ and JL conceived and designed the present study, and revised the manuscript for important intellectual content. All authors read and approved the final version of the manuscript.

\section{Ethics approval and consent to participate}

Not applicable.

\section{Patient consent for publication}

Informed consent was obtained from the patient for the report of the present case.

\section{Competing interests}

The authors declare that they have no competing interests.

\section{References}

1. Assouline-Dayan Y, Chang C, Greenspan A, Shoenfeld Y and Gershwin ME: Pathogenesis and natural history of osteonecrosis. Semin Arthritis Rheum 32: 94-124, 2002.

2. Dubois EL and Cozen L: Avascular (aseptic) bone necrosis associated with systemic lupus erythematosus. JAMA 174: 966-971, 1960.

3. Abu-Shakra M, Buskila D and Shoenfeld Y: Osteonecrosis in patients with SLE. Clin Rev Allergy Immunol 25: 13-24, 2003.

4. Zhang K, Zheng Y, Jia J, Ding J and Wu Z: Systemic lupus erythematosus patients with high disease activity are associated with accelerated incidence of osteonecrosis: A systematic review and meta-analysis. Clin Rheumatol 37: 5-11, 2018.

5. Cavallasca JA, Laborde HA, Araujo MB and Nasswetter GG: Multiple avascular necrosis in a patient with systemic lupus erythematosus/systemic sclerosis overlap syndrome. Clin Rheumatol 24: 406-408, 2005.

6. Darlington LG: Osteonecrosis at multiple sites in a patient with systemic lupus erythematosus. Ann Rheum Dis 44: 65-66, 1985.

7. Fajardo-Hermosillo LD, López-López L, Nadal A and Vilá LM: Multifocal osteonecrosis in systemic lupus erythematosus: Case report and review of the literature. BMJ Case Rep 2013: bcr2013008980, 2013. 
8. Fishel B, Caspi D, Eventov I, Avrahami E and Yaron M: Multiple osteonecrotic lesions in systemic lupus erythematosus. J Rheumatol 14: 601-604, 1987.

9. García-Miguel J, Calvet-Fontova J, Maymó-Guarsch J and Carbonell-Abelló J: Multifocal osteonecrosis in a patient with systemic lupus erythematosus. Med Clin (Barc) 127: 276, 2006 (In Spanish).

10. Greenhagen RM, Crim BE, Shinabarger AB and Burns PR: Bilateral osteonecrosis of the navicular and medial cuneiform in a patient with systemic lupus erythematosus: A case report. Foot Ankle Spec 5: 180-184, 2012.

11. Guillaume MP, Brandelet B and Peretz A: Unusual high frequency of multifocal lesions of osteonecrosis in a young patient with systemic lupus erythematosus. Br J Rheumatol 37: 1248-1249, 1998.

12. Lawal YZ, Ogirima MO, Dahiru IL, Maitama MI, Ejagwulu FS and Abubakar K: Bilateral osteonecrosis of the femoral heads in a patient with systemic lupus erythematosus. Ann Afr Med 10: 64-65, 2011.

13. Outwater E, Oates E and Sarno RC: Bilateral distal tibial osteonecrosis in systemic lupus erythematosus. AJR Am J Roentgenol 152: 895-896, 1989.

14. Salesi M, Karimifar M, Mottaghi P, Sayedbonakdar Z and Karimzadeh H: A case of SLE with bilateral osteonecrosis of femoral heads and bone infarct in distal of femur. Rheumatol Int 30: 527-529, 2010

15. Seymour MW, Mitchell AW and Mackworth-Young CG Bilateral calcaneal osteonecrosis in a patient with systemic lupus erythematosus. Rheumatology (Oxford) 44: 586, 2005.

16. Shimizu M, Tokuhisa Y, Ishikawa S, Ueno K, Yokoyama T and Yachie A: Multiple osteonecrosis in a patient with juvenile systemic lupus erythematosus. J Clin Rheumatol 19: 160, 2013.

17. Bossuyt X, Frans J, Hendrickx A, Godefridis G, Westhovens R and Mariën G: Detection of anti-SSA antibodies by indirect immunofluorescence. Clin Chem 50: 2361-2369, 2004.

18. Abeles M, Urman JD and Rothfield NF: Aseptic necrosis of bone in systemic lupus erythematosus. Relationship to corticosteroid therapy. Arch Intern Med 138: 750-754, 1978.

19. Caramaschi P, Biasi D, Dal Forno I and Adami S: Osteonecrosis in systemic lupus erythematosus: An early, frequent, and not always symptomatic complication. Autoimmune Dis 2012: 725249, 2012.

20. Zizic TM: Osteonecrosis. Curr Opin Rheumatol 3: 481-489, 1991

21. Halland AM, Klemp P, Botes D, Van Heerden BB, Loxton A and Scher AT: Avascular necrosis of the hip in systemic lupus erythematosus: The role of magnetic resonance imaging. Br J Rheumatol 32: 972-976, 1993.

22. Hungerford DS and Zizic TM: II. The treatment of ischemic necrosis of bone in systemic lupus erythematosus. Medicine (Baltimore) 59: 143-148, 1980
23. Ehmke TA, Cherian JJ, Wu ES, Jauregui JJ, Banerjee S and Mont MA: Treatment of osteonecrosis in systemic lupus erythematosus: A review. Curr Rheumatol Rep 16: 441, 2014.

24. Mont MA, Glueck CJ, Pacheco IH, Wang P, Hungerford DS and Petri M: Risk factors for osteonecrosis in systemic lupus erythematosus. J Rheumatol 24: 654-662, 1997.

25. Zhu KK, Xu WD, Pan HF, Zhang M, Ni J, Ge FY and Ye DQ: The risk factors of avascular necrosis in patients with systemic lupus erythematosus: A meta-analysis. Inflammation 37: 1852-1864, 2014.

26. Sayarlioglu M, Yuzbasioglu N, Inanc M, Kamali S, Cefle A, Karaman O, Onat AM, Avan R, Cetin GY, Gul A, et al: Risk factors for avascular bone necrosis in patients with systemic lupus erythematosus. Rheumatol Int 32: 177-182, 2012.

27. Zen M, Canova M, Campana C, Bettio S, Nalotto L, Rampudda M, Ramonda R, Iaccarino L and Doria A: The kaleidoscope of glucorticoid effects on immune system. Autoimmun Rev 10: 305-310, 2011.

28. De Nijs RN: Glucocorticoid-induced osteoporosis: A review on pathophysiology and treatment options. Minerva Med 99: 23-43, 2008.

29. Weldon D: The effects of corticosteroids on bone: Osteonecrosis (avascular necrosis of the bone). Ann Allergy Asthma Immunol 103: 91-97, 2009.

30. Zizic TM, Marcoux C, Hungerford DS, Dansereau JV and Stevens MB: Corticosteroid therapy associated with ischemic necrosis of bone in systemic lupus erythematosus. Am J Med 79: 596-604, 1985

31. Vande Berg BC, Malghem J, Lecouvet FE, Devogelaer JP, Maldague B and Houssiau FA: Fat conversion of femoral marrow in glucocorticoid-treated patients: A cross-sectional and longitudinal study with magnetic resonance imaging. Arthritis Rheum 42: 1405-1411, 1999.

32. Mont MA and Jones LC: Management of osteonecrosis in systemic lupus erythematosus. Rheum Dis Clin North Am 26 279-309, vi, 2000

33. Marker DR, Seyler TM, McGrath MS, Delanois RE, Ulrich SD and Mont MA: Treatment of early stage osteonecrosis of the femoral head. J Bone Joint Surg Am 90 (Suppl 4): S175-S187, 2008.

This work is licensed under a Creative Commons Attribution-NonCommercial-NoDerivatives 4.0 International (CC BY-NC-ND 4.0) License. 